\title{
Real-Life Multimarker Monitoring in Patients with Heart Failure: Continuous Remote Monitoring of Mobility and Patient-Reported Outcomes as Digital End Points in Future Heart-Failure Trials
}

\author{
Frank Kramer ${ }^{a}$ Javed Butler ${ }^{b}$ Sanjiv J. Shah ${ }^{c} \quad$ Christian Jung ${ }^{d}$ Savina Nodarie \\ Stephan Rosenkranz ${ }^{f}$ Michele Senni ${ }^{g} \quad$ Luke Bamber $^{\mathrm{h}} \quad$ Stephan Cichos $^{i}$ Chrysanthi Dori ${ }^{j}$ \\ Toeresin Karakoyun $^{k}$ Gabriele Jenny Köhlerl Kinjal Patel ${ }^{m}$ Paolo Piraino ${ }^{\text {n }}$ \\ Thomas Viethen $^{\circ}$ Praneeth Chennuru ${ }^{p}$ Ayse Paydar ${ }^{q}$ Jason Sims ${ }^{r}$ Richard Clark ${ }^{r}$ \\ Rob van Lummel ${ }^{s} \quad$ Alexandra Müller ${ }^{t} \quad$ Chad Gwaltney $^{\mathrm{u}}$ Salko Smajlovic ${ }^{v}$ \\ Hans-Dirk Düngen ${ }^{w}$ Wilfried Dinh ${ }^{x}$ \\ a Bayer AG, Medical Devices \& eHealth Clinical, Wuppertal, Germany; ${ }^{b}$ Department of Medicine, \\ University of Mississippi Medical Center, Jackson, MS, USA; ${ }^{C}$ Northwestern University, Feinberg \\ School of Medicine, Chicago, IL, USA; ${ }^{d}$ Division of Cardiology, Pulmonary Diseases and Vascular

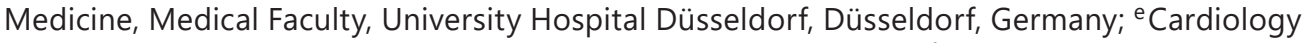 \\ Department, University and Spedali Civili of Brescia, Brescia, Italy; ${ }^{f}$ Department of Cardiology, \\ Heart Center at the University Hospital Cologne and Cologne Cardiovascular Research Center, \\ Cologne, Germany; ${ }^{9}$ Division of Cardiology, Papa Giovanni XXIII Hospital, Bergamo, Italy; ${ }^{\text {h Bayer }}$ \\ AG, Health Economics and Outcomes Research, Wuppertal, Germany; ${ }^{i}$ Bayer AG, Clinical Sciences \\ Data Management, Wuppertal, Germany; ${ }^{j}$ Bayer US, LLC, Bayer HealthCare Pharmaceuticals Inc., \\ Global Clinical Information and Analytics, Data Acquisition Technology Integration, Whippany, NJ, \\ USA; kBayer Business Services GmbH, IT BP Pharmaceuticals, Clinical, Wuppertal, Germany; 'Bayer \\ AG, Translational Studies Operations, Wuppertal, Germany; ${ }^{m}$ Bayer US, LLC, Bayer HealthCare \\ Pharmaceuticals Inc., Global Project Management, Whippany, NJ, USA; ${ }^{n}$ Bayer AG, Research and Early \\ Development Statistics, Berlin, Germany; ${ }^{\circ}$ Bayer AG, Medical Experts Cardiology and Coagulation, \\ Wuppertal, Germany; ${ }^{P}$ Global Customer Success Team, SAP Health, SAP SE, Walldorf, Germany; \\ ISAP AG, SAP Digital Business Services - EMEA, SAP Deutschland SE \& Co. KG, Walldorf, Germany; \\ 'Medtronic, Mounds View, Minneapolis, MN, USA; ${ }^{5}$ McRoberts B.V., The Hague, The Netherlands; \\ ${ }^{t}$ sovanta AG, Heidelberg, Germany; " Gwaltney Consulting, Westerly, RI, USA; ${ }^{v}$ Huawei Technologies \\ Deutschland GmbH, Düsseldorf, Germany; ${ }^{w}$ Medical Department, Division of Cardiology, Charité \\ Campus Virchow Clinic, Berlin University of Medicine, Berlin, Germany; ${ }^{\times}$Bayer AG, Experimental \\ Medicine Cardiovascular/Hematology, Wuppertal, Germany
}

\section{Keywords}

Heart failure $\cdot$ Activity monitors $\cdot$ Patient-reported outcomes $\cdot$ Wearables $\cdot$ Biomarker

\section{Abstract}

Aims: Heart failure (HF) affects approximately 26 million people worldwide. With an aging global population, innovative approaches to $\mathrm{HF}$ evaluation and management are needed to cope with the worsening HF epidemic. The aim of the Real-Life Multimarker Monitoring in 
Patients with Heart Failure (REALIsM-HF) study (NCT03507439) is to evaluate a composite instrument comprising remote, real-time, activity-monitoring devices combined with daily electronic patient-reported outcome (ePRO) items in patients who have been hospitalized for HF and are undergoing standard HF assessment (e.g., 6-min walking distance [6MWD], blood biomarkers, Kansas City Cardiomyopathy Questionnaire [KCCQ], and echocardiography). Methods: REALIsM-HF is an ongoing, 12-week, observational study enrolling 80-100 patients aged $\geq 45$ years with $\mathrm{HF}$ with preserved ejection fraction (HFpEF; EF $\geq 45 \%$ ) or reduced $\mathrm{EF}$ (HFrEF; EF $\leq 35 \%)$. Statistical analyses will include examining the association between data from wearables (the $\mathrm{AVIVO}^{\odot}$ mobile patient management patch or VitalPatch ${ }^{\odot}$ biosensor, and the DynaPort MoveMonitor ${ }^{\odot}$ ), daily ePROs, and conventional HF metrics (e.g., serum/plasma biomarkers, 6MWD, KCCQ, and echocardiographic parameters). The feasibility of and patient compliance with at-home devices will be documented, and the data captured for the purpose of establishing reference values in patients with HFpEF or HFrEF will be summarized. Conclusions: The REALIsM-HF study is to evaluate the longitudinal daily activity profiles of patients with $\mathrm{HF}$ and correlate these with changes in serum/plasma biomarker profiles, symptoms, quality of life, and cardiac function and morphology to inform the use of wearable activity monitors for developing novel therapies and managing patients.

\section{Introduction}

Heart failure (HF) is a global problem that continues to increase in prevalence [1]. Patients with HF have high morbidity and mortality rates, and frequent hospitalization is a major problem [2]. Historically, HF has been classified as having reduced ejection fraction (HFrEF) or preserved EF (HFpEF). Importantly, the prevalence of HFpEF, but not HFrEF, increased significantly from 1987 to 2001 [3].

Although the benefits of pharmacotherapy in HFrEF have been shown in several studies, there is no clear evidence that angiotensin-converting enzyme inhibitors, mineralocorticoid receptor antagonists, or $\beta$-blockers are effective in the treatment of HFpEF [4-7]. In the Treatment of Preserved Cardiac Function Heart Failure with an Aldosterone Antagonist (TOPCAT) study, spironolactone did not significantly reduce the incidence of cardiovascular death, aborted cardiac arrest, or hospitalization in patients with HFpEF [5]. In addition, there are several retrospective analyses that have shown that standard therapies do not work in this subpopulation $[8,9]$. Despite rapid initial stabilization of symptoms following treatment with diuretics, patients with HF often remain vulnerable to further adverse cardiac events during the lead-up to hospitalization and also early postdischarge. Consequently, novel objective markers are needed to supplement or improve our current limited ability to predict impending adverse outcomes and serve as potential new end points for HFpEF.

Drawing meaningful conclusions from clinical trials relies on the accurate assessment of changes in clinical parameters. Monitoring of daily activities may be a better, more objective measure of clinical status in the elderly than a physician's assessment of New York Heart Association (NYHA) class; however, current monitoring of patients with HF is restricted to single point-in-time measurements or limited periods of continuous monitoring during hospitalization. These measurements may not reflect the cardiovascular (CV) capacity restrictions that can occur in daily life, particularly in HFpEF during physical activity; however, technologies are available that facilitate continuous remote monitoring of activity and CV data under real-life conditions. Measuring physical activity using accelerometers worn by patients with HF has been reported previously [10].

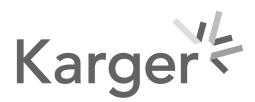




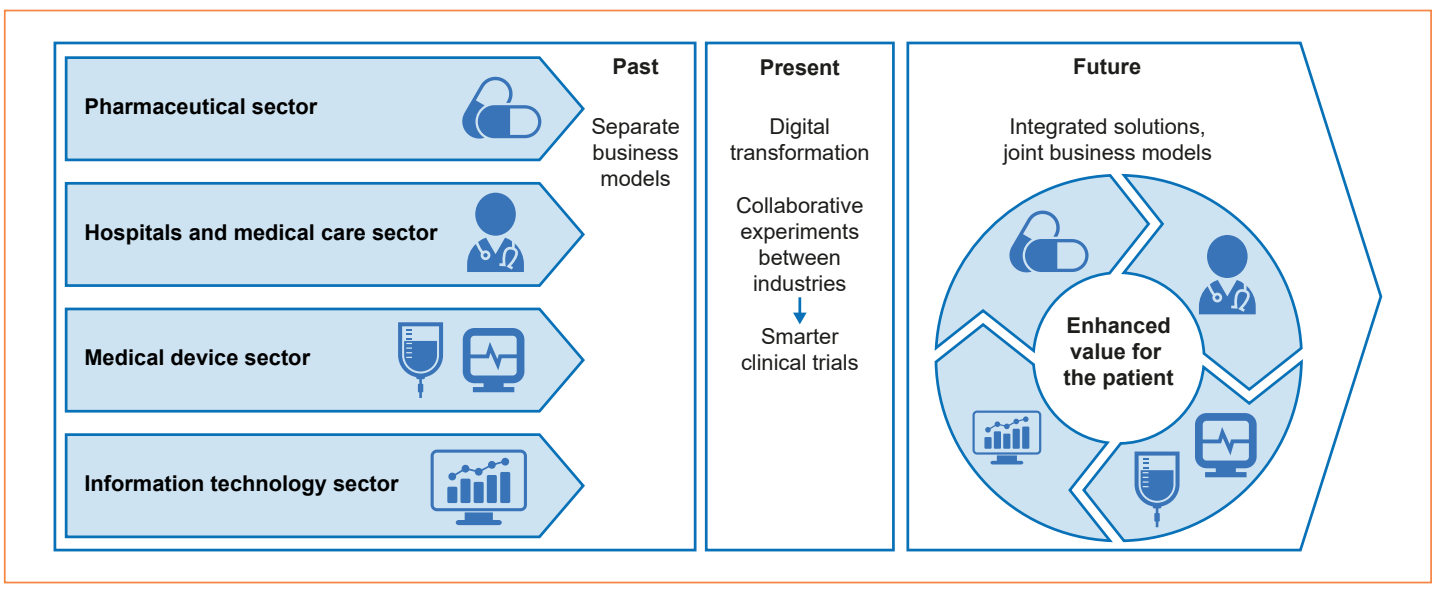

Fig. 1. REALIsM-HF study: a template for future clinical studies. A collaborative experiment paving the way to integrated health solutions for patients with HF by engaging diverse health care and industry sectors. HF, heart failure.

In patients with HF, the most consistently reported problems that affect quality of life (QoL) are limitations to physical activity and mobility [11]. Innovative approaches to measuring physical activity in relation to the patient's perception of symptoms could aid the development of new treatments [12]. Patient-reported outcomes (PROs), which describe the patient's experience of living with HF, are generally captured by questionnaires. However, with certain exceptions (e.g., the Kansas City Cardiomyopathy Questionnaire [KCCQ]), the questionnaires available for measuring physical activity in HF were not developed according to the relevant US Food and Drug Administration (FDA) guidelines [13] or specifically for patients with HFpEF. PROs may complement objective clinical measures of daily physical activity by providing a subjective assessment of symptom burden (e.g., fatigue or dyspnea on exertion) experienced by the patient in achieving daily physical activity thresholds, and the relevance to the patient of changes in physical functioning.

Collecting data from HFpEF-specific PRO questionnaires and physical activity monitoring and correlating each with conventional end points and biomarkers hold promise for the identification of potential new end points for future trials, by providing objective data on disease progression and forewarning of impending adverse events. The Real-Life Multimarker Monitoring in Patients with Heart Failure (REALIsM-HF) study described here represents a novel partnership entailing collaboration across the traditional borders between academia and diverse industrial disciplines (Fig. 1).

\section{Aims}

The aim of the REALIsM-HF (NCT03507439) pilot study is to evaluate a composite instrument combining remote physical activity monitoring with daily electronic PRO questionnaires in patients with HFpEF and HFrEF under real-life conditions. The study will also start building reference data for use in future drug and device development programs as well as in the clinical management of patients. Although this study is intended primarily for patients with HFpEF, its HFrEF data will help to generate hypotheses and aid in determining the feasibility of physical activity monitoring with different devices, both in patients with HFpEF and those with HFrEF. Associations will be explored between clinically relevant changes in the data recorded by each device and conventional end points such as the 6-min walking distance (6MWD), real-life gait analysis, biomarkers, and clinical events, with the 
Table 1. Primary and secondary objectives of the REALIsM-HF study

\section{Primary objective}

- $\quad$ Measure and quantify daily physical activity in patients with HFpEF and HFrEF under real-life conditions using the AVIVO MPM patch or VitalPatch, and DynaPort MoveMonitor

\section{Secondary objectives}

- $\quad$ Evaluate the association between data from the AVIVO MPM patch/VitalPatch/DynaPort Move-

Monitor and adverse event/clinical outcome data from during the study period

- $\quad$ Assess the baseline level, changes over time and variability in tissue impedance, heart rate, respiratory rate, and EKG-derived parameters, and assess the average person-to-person physiological variability and within-patient standard deviation

- $\quad$ Assess the feasibility of physical activity tracking with the AVIVO MPM patch or VitalPatch, and DynaPort MoveMonitor in patients with HF

- $\quad$ Correlate physical activity data from the AVIVO MPM patch or VitalPatch, and DynaPort MoveMonitor

- $\quad$ Evaluate the relationship of physical activity levels, tissue impedance, heart rate, respiratory rate, and EKG-derived parameters with important measures in HFpEF, such as QoL, PROs, biomarkers, and clinical outcomes (e.g., endpoints such as hospitalization for HF, cardiovascular death, emergency visits)

- $\quad$ Support the development of HFpEF-specific questions (PROs) for cross-sectional and longitudinal testing/validating

- $\quad$ Determine the variability of device-derived biomarkers between and within patients over time, and validate the use of suitable biomarkers for clinical use in the context of $\mathrm{HF}$

- Explore longitudinal changes in transcutaneous thoracic impedance measurement (by the AVIVO MPM patch) as a surrogate for fluid status in patients with acute HF and after stabilization

- $\quad$ Evaluate hs-copeptin as a potential biomarker in HF (as a surrogate marker for arginine vasopressin)

- $\quad$ Correlate blood-based biomarkers of congestion (e.g., NTproBNP/hs-copeptin) with a functional marker (e.g., impedance; AVIVO MPM patch)

- $\quad$ Correlate data collected from the AVIVO MPM patch/VitalPatch and inpatient clinical data collected during hospital stays (e.g., echocardiography, weight gain/loss, NYHA class, and heart rate)

- $\quad$ Explore the relationship between physical activity, 6MWD, PROs, biomarkers, and adverse events/ clinical outcomes

- $\quad$ Investigate the accuracy of patients' self-reports (PROs and KCCQ) of time spent undertaking physical activity in real life vs. objective assessment by the AVIVO MPM patch or VitalPatch, and DynaPort MoveMonitor

6MWD, 6-min walking distance; EKG, electrocardiogram; HF, heart failure; HFpEF, HF with preserved ejection fraction; HFrEF, HF with reduced ejection fraction; hs-copeptin, high-sensitivity copeptin; KCCQ, Kansas City Cardiomyopathy Questionnaire; MPM, Mobile Patient Management; NTproBNP, N-terminal pro-brain natriuretic peptide; NYHA, New York Heart Association; PRO, patient-reported outcome; QoL, quality of life; REALIsM-HF, Real-Life Multimarker Monitoring in Patients with Heart Failure.

intention of generating evidence for the definition of novel clinical end points that can be used for both drug registration and the management of patients.

Measuring objective and subjective data by means of device-based activity monitoring and electronic PROs (ePROs), respectively, will capture all relevant aspects of physical activity in patients with HFpEF. Given the multiplicity of parameters and wealth of data, the aim is to develop a robust, precisely predictive instrument that is highly correlated to the physiological responses in patients with HFpEF by combining data on physical activity, digital, and laboratory biomarkers.

\section{Study Design}

The REALIsM-HF study is a nonrandomized, multicenter, 12-week, observational, prospective patient study. 
Table 2. REALIsM-HF study inclusion and exclusion criteria

\section{Inclusion criteria}

Worsening HF requiring hospitalization within the past $72 \mathrm{~h}$ for the initiation of intensification of HF therapy with at least 1 of the following:

- BNP $\geq 100 \mathrm{pg} / \mathrm{mL}$ or NTproBNP $\geq 400 \mathrm{pg} / \mathrm{mL}$ (sinus rhythm) or BNP $\geq 300 \mathrm{pg} / \mathrm{mL}$ or

NTproBNP $\geq 1,200 \mathrm{pg} / \mathrm{mL}$ (atrial fibrillation)

- radiographic evidence of pulmonary congestion

- catheterization-documented elevated filling pressures at rest or with exercise

\section{For HFrEF only:}

- EF $\leq 35 \%$ during the 3 months before study inclusion

\section{For HFpEF only:}

- $\mathrm{EF} \geq 45 \%$ during the 3 months before study inclusion

- a willingness and ability to wear the AVIVO MPM patch or VitalPatch, and DynaPort MoveMonitor during the trial

\section{Exclusion criteria}

- Inability to comply with planned study requirements

- Hemoglobin $<10.0 \mathrm{~g} / \mathrm{dL}$

- Acute coronary syndrome or PCI during the 3 months of informed consent

- Listing for heart transplantation and/or anticipated implantation of a ventricular assist device

- Inability to exercise or dependence on supplemental oxygen

- Known clinically significant persistent coronary ischemia

- A major cardiovascular event or surgical procedure during the 3 months before informed consent

- PCI, CABG, or implantation of a CRTD planned between randomization and visit 4

- Presence of implantable devices with active minute ventilation sensors

- Severe uncorrected valvular heart disease or known clinically relevant ventricular arrhythmias

- Severe pulmonary disease

- Heavy alcohol consumption or the use of illicit drugs

- Regular (more than once per week) participation in swimming or water aerobics

- Active myocarditis, primary hypertrophic cardiomyopathy, or constrictive pericarditis or pericardial tamponade

BNP, brain natriuretic peptide; CABG, coronary artery bypass graft; CRTD, cardiac resynchronization therapy implantable cardioverter defibrillator; EF, ejection fraction; HF, heart failure; HFpEF, HF with preserved EF; HFrEF, HF with reduced EF; MPM, Mobile Patient Management; NTproBNP, N-terminal pro-brain natriuretic peptide; PCI, percutaneous coronary intervention; REALIsM-HF, Real-Life Multimarker Monitoring in Patients with Heart Failure.

\section{Objectives}

The primary objective of the REALIsM-HF study is to measure and quantify daily physical activity in patients with HFpEF and HFrEF following hospitalization for HF under real-life conditions using 3 wearable monitoring devices. The secondary objectives are to: correlate biometric monitoring data with established measures of safety, clinical outcomes, and inpatient data obtained during hospitalization and possible rehospitalization; compare and correlate data obtained from the 3 monitoring devices; support the development of HFpEFspecific PROs and investigate their accuracy by comparison with objective physical activity data; and evaluate a meaningful change in physical activity in an HFpEF patient population. Table 1 presents a complete list of the study objectives.

\section{Study Population}

Male or female patients aged $\geq 45$ years with an established diagnosis of HF with NYHA class II-IV symptoms and hospitalized owing to worsening symptoms will be recruited in Europe and the USA. Table 2 describes inclusion and exclusion criteria. A total of 80-100 patients, $60-70$ with $\mathrm{HFpEF}$ ( $\mathrm{EF} \geq 45 \%$ ) and 20-30 with $\mathrm{HFrEF}$ (EF $\leq 35 \%$ ), are required to complete all elements of the study. Recruitment is based on similar studies assessing physical activity and the expected variability in measurements taken under unstandardized conditions [14].

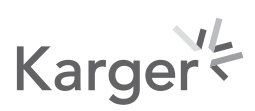




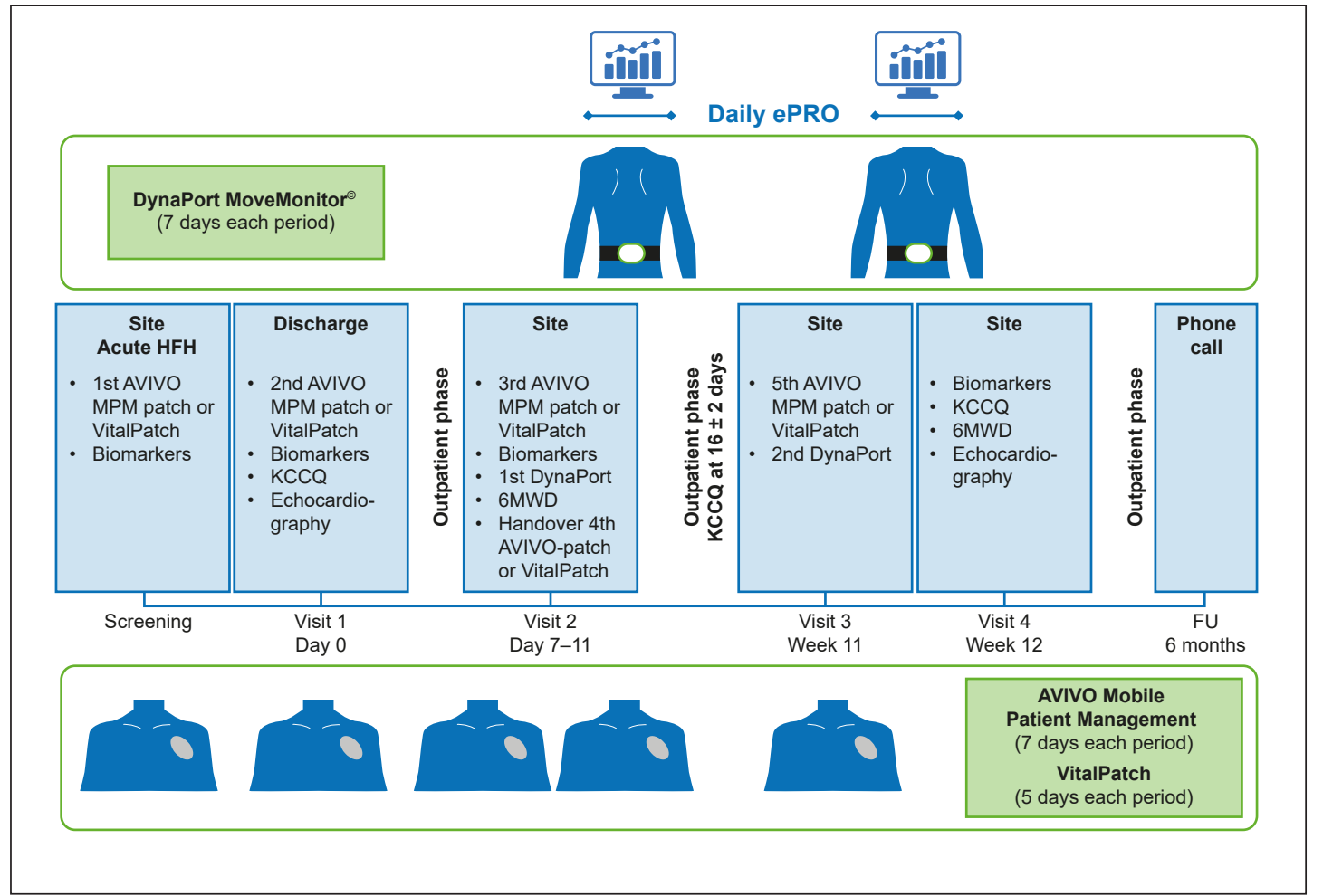

Fig. 2. REALIsM-HF study: study design. 6MWD, 6-min walking distance; EKG, electrocardiogram; ePRO, electronic patient-reported outcome; FU, follow-up visit; $\mathrm{HF}$, heart failure; $\mathrm{HFH}$, heart failure hospitalization; KCCQ, Kansas City Cardiomyopathy Questionnaire; MPM, Mobile Patient Management; REALIsM-HF, RealLife Multimarker Monitoring in Patients with Heart Failure.

\section{Study Procedures}

Table 3 presents a summary of study procedures. Within $72 \mathrm{~h}$ of hospitalization, patients will be asked if they wish to participate in the study. All enrolled patients will receive the $\mathrm{AVIVO}^{\odot}$ mobile patient management (MPM) patch (Medtronic, Minneapolis, MN, USA) or VitalPatch $^{\odot}$ biosensor (VitalConnect, San José, CA, USA), and the DynaPort MoveMonitor ${ }^{\odot}$ (McRoberts BV, The Hague, The Netherlands), and training in their use will be provided (note: the AVIVO MPM patch became commercially unavailable after supplying approximately 10 patients, and the VitalPatch was selected as a suitable replacement). All devices are approved for use in the EU (CE-marked) and in the USA (FDA-approved). Selection of the devices was based on a systematic landscape analysis of available devices. Selection criteria included the approval of devices, the number of vital signs that they monitor, and the quality of data that they generate [15]. Data are collected continuously by each device and periodically transmitted to dedicated servers for analysis. The AVIVO MPM patch monitoring system will be worn for 5 separate 7-day monitoring periods (5 patches/patient in total). As an alternative to the AVIVO MPM patch, the VitalPatch biosensor will be worn for 5 separate 5-day monitoring periods ( 5 patches/patient in total). The AVIVO MPM patch and VitalPatch are wireless, peel-and-stick patient-monitoring devices designed to have a minimal impact on the patient's daily life. The AVIVO MPM patch continuously measures and records physiological data, including electrocardiogram (EKG) and EKG-derived parameters (e.g., heart rate, heart rate variability, atrial fibrillation burden, and arrhythmias), respiratory rate, fluid status via local transcutaneous thoracic impedance, physical activity (duration and intensity), and posture. 


\section{Digital Biomarkers}

\begin{tabular}{|c|c|}
\hline \multicolumn{2}{|l|}{ Digit Biomark 2020;4:45-59 } \\
\hline DOI: $10.1159 / 000507696$ & $\begin{array}{l}\text { (c) } 2020 \text { The Author(s). Published by S. Karger AG, Basel } \\
\text { www.karger.com/dib }\end{array}$ \\
\hline
\end{tabular}

Kramer et al.: Mobility and ePRO Monitoring in HF

Table 3. Overview of study procedures and variables in the REALIsM-HF study

\begin{tabular}{|c|c|c|c|c|c|c|c|c|}
\hline \multirow{2}{*}{$\begin{array}{l}\text { Study period } \\
\text { Visit No. }\end{array}$} & \multicolumn{2}{|c|}{ Hospitalization phase } & \multicolumn{5}{|c|}{ Outpatient phase } & \multirow{2}{*}{$\begin{array}{l}\text { Safety FU call } \\
5\end{array}$} \\
\hline & Screening ${ }^{\mathrm{a}}$ & 1 & 2 & & & 3 & 4 & \\
\hline \multirow[t]{2}{*}{ Visit type } & \multicolumn{2}{|c|}{ on site } & \multicolumn{3}{|l|}{ on site } & on site & on site & telephone \\
\hline & $\begin{array}{l}\text { screening } \\
\text { assessment }\end{array}$ & $\begin{array}{l}\text { at hospital } \\
\text { discharge }\end{array}$ & & & & & & \\
\hline Week after discharge & & & 1 & 2 & 3 & 11 & 12 & \\
\hline Day and allowed deviations & & 0 & $9 \pm 2$ & $6 \pm 2$ & $23 \pm 2$ & $77 \pm 2$ & $84 \pm 2$ & $\begin{array}{l}6 \text { months } \pm \\
4 \text { weeks }\end{array}$ \\
\hline Signed informed consent form & $\checkmark$ & & & & & & & \\
\hline Inclusion/exclusion criteria & $\checkmark$ & & & & & & & \\
\hline KCCQ & & $\checkmark$ & & $\checkmark$ & & & $\checkmark$ & \\
\hline Daily ePRO & & & $>-1 \mathrm{st}-<$ & & & $>-2 \mathrm{r}$ & & \\
\hline Weekly ePRO & & & & $\checkmark$ & & & $\checkmark$ & \\
\hline Demographic data & $\checkmark$ & & & & & & & \\
\hline Medical and surgical history & $\checkmark$ & & & & & & & \\
\hline Physical examination & $\checkmark$ & $\checkmark$ & $\checkmark$ & & & $\checkmark$ & $\checkmark$ & \\
\hline Height & $\checkmark$ & & & & & & & \\
\hline Weight & $\checkmark$ & $\checkmark$ & $\checkmark$ & & & & $\checkmark$ & \\
\hline 12-lead EKG & $\checkmark$ & $\checkmark$ & & & & & $\checkmark$ & \\
\hline NYHA class & $\checkmark$ & $\checkmark$ & $\checkmark$ & & & & $\checkmark$ & \\
\hline Blood pressure and heart rate & $\checkmark$ & $\checkmark$ & $\checkmark$ & & & $\checkmark$ & $\checkmark$ & \\
\hline Adverse events & $\checkmark$ & $\checkmark$ & $\checkmark$ & & & $\checkmark$ & $\checkmark$ & $\checkmark$ \\
\hline Concomitant medication & $\checkmark$ & $\checkmark$ & $\checkmark$ & & & $\checkmark$ & $\checkmark$ & $\checkmark$ \\
\hline Echocardiography & & $\checkmark$ & & & & & $\checkmark$ & \\
\hline 6MWD & & & $\checkmark$ & & & & $\checkmark$ & \\
\hline $\begin{array}{l}\text { DynaPort MoveMonitor (for } 7 \text { days) } \\
>\text { start < end }\end{array}$ & & & $>-1 \mathrm{st}-<$ & & & $>-2 n$ & & \\
\hline $\begin{array}{l}\text { AVIVO MPM patch (for } 7 \text { days) or Vital- } \\
\text { Patch (for } 5 \text { days) }{ }^{c}>\text { start <end }\end{array}$ & $>-1$ st $-<$ & $>-2$ nd $-<$ & $>-3 r d-<$ & $>-4$ th $-<$ & & $>-5 \mathrm{t}$ & & \\
\hline Blood sample for biomarkers ${ }^{\mathrm{d}}$ & $\checkmark$ & $\checkmark$ & $\checkmark$ & & & & $\checkmark$ & \\
\hline
\end{tabular}

6MWD, 6-min walking distance; EKG, electrocardiogram; ePRO, electronic patient-reported outcome; FU, follow-up; Gal-3, galectin-3; GDF15, growth/differentiation factor 15; hs-copeptin, high-sensitivity copeptin; hsTnT, high-sensitivity troponin T; IGFBP7, insulin-like growth factor-binding protein 7; KCCQ Kansas City Cardiomyopathy Questionnaire; MPM, Mobile Patient Management; NTproBNP, N-terminal pro-brain natriuretic peptide; NYHA, New York Heart Association; REALIsM-HF, Real-Life Multimarker Monitoring in Patients with Heart Failure; sST2, soluble suppression of tumorigenicity 2.

${ }^{\mathrm{a}}$ Within $72 \mathrm{~h}$ of hospitalization.

${ }^{\mathrm{b}}$ ePRO is completed daily/weekly during the AVIVO MPM patch or VitalPatch, and DynaPort MoveMonitor in the parallel monitoring period.

${ }^{\mathrm{c}}$ DynaPort MoveMonitor monitoring periods: week 2 after hospital discharge (days 7-14) and weeks 11-12 (days 77-84). AVIVO patch monitoring periods: $\leq 7$ days during the hospital stay, week 1 after hospital discharge (days 0-7), during weeks 2 and 3 (days 7-14, days 17-24), and weeks 11-12 (days 77-84). VitalPatch monitoring periods: $\leq 5$ days during the hospital stay, week 1 after hospital discharge (days 0-5), during weeks 2 and 3 (days $7-12$, days 17-22), and weeks 11-12 (days 77-82).

${ }^{\mathrm{d}}$ Central laboratory assessments: NTproBNP, hs-copeptin, hsTnT; optional: GDF15, IGFBP7, sST2, Gal-3. 
The VitalPatch continuously measures and stores EKG and EKG-derived parameters, respiration rate, skin temperature activity, posture, and activity. The DynaPort MoveMonitor will be worn on the lower back for 2 separate 7-day periods, in parallel with the AVIVO MPM patch or VitalPatch (Fig. 2), to monitor various parameters, including the distribution of different types of physical activity (lying, sitting, standing, walking, shuffling, stair walking, and cycling). Additional parameters obtained from the DynaPort MoveMonitor include energy expenditure (light, moderate, and vigorous activity) and sleep movements. A minimum of 5 days of observation is required to obtain reproducible results in all categories [16].

\section{QoL Assessments/PROs}

The KCCQ is a validated and widely used patient-reported HF health status measure comprising a 23-item questionnaire that independently measures the impact on the patient of HF and its treatment in 7 distinct domains [17]. The KCCQ will be performed at hospital discharge, and at weeks 2 and 12; at each time point, the overall summary score will be documented together with the domain scores, with a focus on the physical limitation, social limitation, and symptom domains (frequency and burden).

Study participants will be asked to record their symptoms and physical activity by completing a daily ePRO diary (after 18:00) during each period of physical activity monitoring (Table 3; weeks 1-2 and weeks 11-12). Questions in the daily ePRO diary relate to 3 areas: the amount and type of physical activity undertaken in the previous $24 \mathrm{~h}$ (e.g., walking inside and outside the home, housework, and climbing stairs), the severity of key HF symptoms (e.g., fatigue, dyspnea, and peripheral edema), and overall perceptions of the amount of activity and symptoms severity. After 7 days of capturing daily ePROs, patients will complete a weekly questionnaire to record their perception of change in both HF symptoms and physical activity since being discharged from hospital. The relationship between the movement data from the activity monitors and scores on the diary items will be examined. The diary data will be used when estimating a threshold for meaningful within-patient change on the activity monitor end points.

\section{Data Collection}

Responses to KCCQ and exploratory daily ePRO diary questions will be collected electronically via a tablet computer (Huawei MediaPad M5, Shenzhen, China) to facilitate regular, accurate patient assessment of daily symptoms and their impact on physical activity, and to minimize recall bias; data will be transferred to the SAP Health for Patient Engagement (SHPE) cloud storage (SAP AG, Walldorf, Germany). The application hosting the questionnaires (sovanta AG, Heidelberg, Germany) was developed and tested by taking into consideration the age of the target patient population and their potential lack of experience with mobile devices (e.g., clear and intuitive instructions, use of a large font). The MediaPads will be preconfigured to disable application installations and will be secured by a patient-specific personal identification number. During enrollment, each patient will receive a MediaPad that is QR-code-linked to a unique patient identification ("Subject ID") on the SHPE cloud storage. The MediaPad will be Wi-Fi and Long-Term Evolution (a 4G mobile communications standard) enabled in order to allow continuous connection to the SHPE cloud service package, thereby allowing reminders and encouragement to complete the questionnaire and data upload; compliance will be assessed via the SHPE. Domain-specific and total KCCQ scores will be computed in the SHPE for each KCCQ assessment before being sent to the data-integration platform for dedicated analysis (see below).

Although there are no specific procedures in place to ensure longitudinal compliance, some countries (e.g., Germany and the USA) operate a renumeration system for investigation site visits, where travel and subsidence costs are met up to a predefined value. In other countries (e.g., Italy), compensation is prohibited by law.

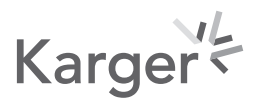




\section{Digital Biomarkers}

\begin{tabular}{|c|c|}
\hline Digit Biomark 2020;4:45-59 & \\
\hline DOI: 10.1159/000507696 & $\begin{array}{l}\text { (c) } 2020 \text { The Author(s). Published by S. Karger AG, Basel } \\
\text { www.karger.com/dib }\end{array}$ \\
\hline
\end{tabular}

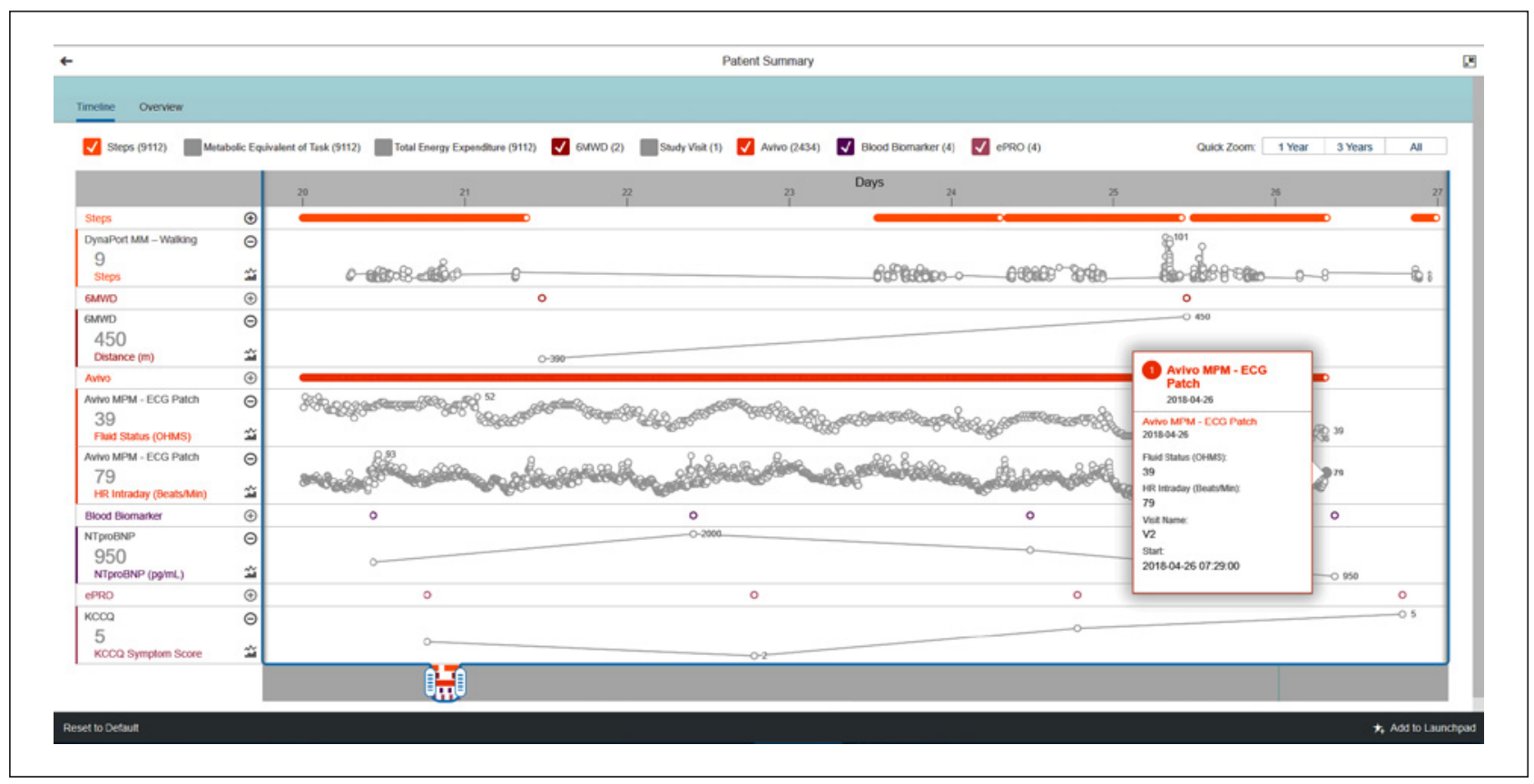

Fig. 3. SAP Medical Research Insights. Screenshot of the SAP Medical Research Insights dashboard displaying example data from a single patient (digital biomarkers from wearables, molecular biomarkers from central laboratory, remotely captured ePRO, and clinical site-measured 6MWD). Orange horizontal bars show continuous or high-frequency measurements, purple circles show point-in-time measurements, gray lines and circles display measurement changes over time. The mouse-flyover box shows detailed data at a single time point and the dashboard can be tailored to display different study parameters. 6MWD, 6-min walking distance; ECG, electrocardiography; ePRO, electronic patient-reported outcome; HR, heart rate; KCCQ, Kansas City Cardiomyopathy Questionnaire; MM, MoveMonitor; MPM, Mobile Patient Management; NTproBNP, Nterminal pro-brain natriuretic peptide.

Data collection and analysis will also encompass health care utilization costs, including, for example, cardiac rehabilitation; however, because such data reflect the real-life behavior of patients in the study, they are not intended to inform the study outcomes.

Following the data analysis at the end of this observational study, results will be distributed to the individual investigation sites.

\section{Data-Integration Platform}

In the REALIsM-HF study, data processing is provided by the SAP Connected Health Platform (CHP). Activity scores from devices, ePROs, computed scores, and other clinical measurements collected during the study are securely transferred to the CHP on a scheduled basis, and are then consolidated and checked for corrupt or inaccurate data. The Subject ID is used to link all data points across the data sources; no identifiable information is stored.

The CHP also offers a default analytical application allowing investigators to visualize, interpret, and draw conclusions from the datasets, and perform further near-real-time statistical analyses if necessary (Fig. 3). 
6MWD Test

A 6MWD test will be performed at week 1 and at weeks 11-12 (Fig. 2). Although the 6MWD test is sometimes used in clinical practice and as an end point in clinical trials and is also the basis of function-based prognostic assessments [18], inconsistencies when administering it may inadvertently diminish the reliability of the results [19]. In the REALIsM-HF study, the 6MWD test will be performed in accordance with American Thoracic Society guidelines [20].

\section{Laboratory Parameters}

In addition to assessing clinical variables, HF status will be characterized at baseline and longitudinally by monitoring serum/plasma biomarkers including high-sensitivity troponin $\mathrm{T}$, galectin-3, growth differentiation factor, soluble suppression of tumorigenicity 2, N-terminal pro-brain natriuretic peptide (NTproBNP), high-sensitivity copeptin, and insulin growth factor-binding protein 7 (online suppl. Table 1; for all online suppl. material, see www.karger.com/doi/10.1159/000507696) [21-38]. Measuring biomarker combinations representing different pathways provides incremental values for risk stratification, and the data generated are expected to act as a reference for future studies. Conversely, established biomarkers, such as NTproBNP, may serve as a reference when evaluating data from the AVIVO MPM patch, VitalPatch, and DynaPort MoveMonitor. Biomarkers will be investigated during the hospital stay and at weeks 1 and 12 after discharge (visits 2 and 4, respectively).

\section{Adverse Events}

All adverse events will be recorded. An adverse event is any untoward medical occurrence, and will be categorized according to its seriousness (resulting in death, is life-threatening, requiring hospitalization or prolongation of hospitalization, or resulting in significant disability or incapacity) and intensity, i.e., mild (transient; minimal intervention), moderate (alleviated with additional specific intervention), or severe (requiring intensive intervention), and causal relationship to the device(s).

\section{Statistical Analyses}

REALIsM-HF is an observational pilot study with multiple objectives, that aims to generate rather than test predefined hypotheses. According to the study objectives, statistical analyses will be descriptive and exploratory in nature. Owing to the multiplicity of parameters being assessed and the repetition of measures over time, it is expected that a high volume of data will be generated for each patient; the number of days of assessment and the total population size are based on simulations performed using a preliminary data set, and have been set to accommodate the study objectives. Statistical methods will include the use of descriptive statistics for summarizing continuous and categorical variables (absolute value and change from baseline per analysis time point, as applicable, and absolute and relative frequencies). Univariate models (e.g., mixed effects) will be used for the assessment of the variability and association of parameters with events (including, but not limited to EF as a stratification factor), multivariate regression models and dimension reduction techniques will be used for the assessment of the correlation between variables from different sources, and clustering techniques will be used to identify subgroups of patients with similar characteristics. 


\section{Discussion}

REALIsM-HF was conceived with innovation as a central pillar of the study design. This approach, while still relatively underused, has been promoted by representatives such as the outgoing FDA Commissioner as the model that should be considered when designing future clinical trials [39].

New approaches are needed to improve signs, symptoms, and functional capacity in patients with HF and reduce mortality and rehospitalization, especially for those with HFpEF. The aim of the REALIsM-HF study is to collect continuous functional, self-reported symptoms, and QoL and laboratory test data in a diverse population of patients with HF, and then define the challenges and test the feasibility of this approach under real-life conditions. Furthermore, given that most patients with HF are elderly, such instruments may help us to understand and evaluate the overlap between the symptoms of HF and frailty (e.g., fatigue, weakness, cachexia, and depression).

The observational study design deployed for REALIsM-HF is suitable for the collection of real-life physical activity data because no treatment is being evaluated, and the statistical analyses are exploratory. Physical activity data are monitored and measured objectively and are unlikely to be subject to investigator- or patient-induced bias [16]. The high density of data generated by continuous data capture, combined with careful consideration of the trial design and patient inclusion criteria, may allow for a smaller patient population than with conventional trials in which only point-in-time measurements can be performed [40].

Participant selection criteria includes those with HFpEF (EF $\geq 45 \%$ ) and HFrEF (EF $\leq 35 \%$ ). HF with midrange EF (HFmrEF; EF 40-49\%) has been recognized [41]; it includes the $\geq 45 \%$ threshold used for HFpEF in this study. The term HFmrEF is relatively new and is not yet well established compared with HFrEF and HFpEF. Indeed, some publications use the term $\mathrm{mEF}$ for patients with missing (unknown) EF, so there is potential for the term HFmrEF to be misinterpreted. Current guidelines assume that identifying patients with HFmrEF as a separate group will stimulate research into the underlying characteristics, pathophysiology, and treatment of this group, even though it represents a gray area between HFrEF and HFpEF. By choosing a cut-off value of $45 \%$ for the HFpEF group in REALIsM-HF, there is a high probability of including only patients with true HFpEF (who might also have subtle abnormalities of systolic function) and not those with HFrEF (which is typically defined as EF <40\%). Notably, patients with a consistently high EF of 40-55\% do not show a statistically significant difference in major outcomes (death, left ventricular assistant device implantation, or transplantation) when compared with patients with HFpEF with an EF > 55\% [42].

Moreover, inclusion of the hospitalization and outpatient phases will facilitate the longitudinal evaluation of changes in daily activity in patients with HF and allow for tracking of rehospitalization rates, a metric that is increasingly used as an outcome measure in clinical trials; up to $30 \%$ of patients with HF are readmitted within 60-90 days of discharge [43]. Continuous remote monitoring of patients under real-life conditions is becoming recognized as a valuable tool for developing new therapeutic agents. Multisensor monitors have been used previously to develop an HF decompensation prediction algorithm in patients with HF, and to monitor changes in fluid status in patients with chronic kidney disease [44]. With a similar goal to that of the REALIsM-HF study, the PROactive consortium has successfully used a combination of disease-specific PRO and activity-monitoring data to assess physical activity in patients with chronic obstructive pulmonary disease (COPD), resulting in its acceptance by the European Medicines Agency as a metric in the development of future COPD therapies [15]. In addition, the aim of the ongoing European Commission-funded Innovative Medicines Initiative "Linking digital assessment of mobility to clinical endpoints to support regulatory acceptance and clinical practice" is to demonstrate that digital detection of low mobility can

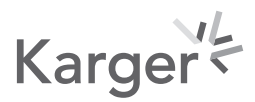


predict clinically relevant outcomes in chronically ill or frail populations [45]. Our ability to collect and manipulate high volumes of data heralds a new era in the remote monitoring of at-risk patients. Devices such as the AVIVO MPM patch, VitalPatch, and DynaPort MoveMonitor are discreet and unobtrusive, and they provide a wealth of data with a minimal impact on the patient. The large volumes of data generated by such devices and ePROs can be collected and analyzed via integrated, applied information technology solutions. The perceived future benefits for patients and practitioners include the real-time monitoring of important CV parameters, treatment adjustments to meet ongoing needs, and understanding patient compliance with treatment recommendations.

This pilot study comprises a patient population with diverse disease characteristics, ages, baseline activity levels, cognitive status, and compliance with completion of the ePRO. The investigations will therefore help to build a set of real-world reference data based on differing behaviors and potentially diverse result patterns across the disease spectrum, that can then be used to help tailor future interventional trials, e.g., through appropriate sample size calculations or improved logistics. These data will therefore be used to inform more effective drug development and personalized treatment options in a population of patients with great medical need, particularly those with HFpEF.

In summary, it is anticipated that the REALIsM-HF study will aid the identification of potential new end points for future HF trials, especially for HFpEF, by exploring the complex relationships between clinically relevant changes in physical activity data from remote monitoring devices, information from PROs, and correlations/associations with conventional end points and biomarkers. The aim is to provide a comprehensive understanding of the clinical utility of wearable devices and how these can be used in conjunction with ePRO questionnaires to capture all aspects of physical activity in a heterogenous population of patients with HFpEF.

Studies such as REALIsM-HF may foster future collaborations between multiple stakeholders to develop new health care approaches. It is hoped that such patient-centered collaborations will help to improve well-being, day-to-day functioning, and QoL as well as reduce morbidity and mortality in this vulnerable patient population.

\section{Acknowledgment}

Medical writing support was provided by Dr Jim Purvis of Oxford PharmaGenesis, Oxford, UK, with funding from Bayer AG. The authors appreciate the contributions made by Chris Economos and the PhysIQ team based in Chicago, IL, USA.

\section{Statement of Ethics}

The study was performed according to the guiding principles detailed in the Declaration of Helsinki and in keeping with applicable local laws and regulations.

\section{Disclosure Statement}

L.B., S.C., W.D., C.D., T.K., G.J.K., F.K., K.P., P.P., and T.V. are employees of Bayer and may hold shares or share options. J.B. received research support from the European Union and National Institutes of Health, Patient-Centered Outcomes Research Institute and serves as a consultant for Amgen, Array, AstraZeneca, Bayer, Boehringer Ingelheim, Bristol-Myers

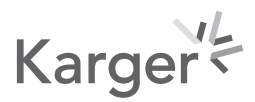


Squibb, CVRx, G3 Pharmaceuticals, Innolife, Janssen, Luitpold, Medtronic, Merck, Novartis, Relypsa, scPharmaceuticals, Stealth Peptides, Vifor Pharma, and ZS Pharma. P.C. and A.P. are employees of SAP Health, SAP SE. R.C. is a former employee of and shareholder in Medtronic, and has had consulting engagements with ARCA Biopharma, Espero Biopharma, MediMergent, and Medtronic. C.G. is an employee of Gwaltney Consulting. A.M. is an employee of sovanta AG. S.N. has no relationships with the industry. S.R. received research support from the Deutsche Forschungsgemeinschaft (DFG) and the Bundesministerium für Bildung und Forschung (BMBF), and speaker fees and serves as a consultant for Abbott, Actelion, Bayer, Bristol-Myers Squibb, Merck Sharp \& Dohme, Novartis, Pfizer, United Therapeutics, and Vifor Pharma; his institution has received research grants from Actelion, Bayer, Novartis, Pfizer, and United Therapeutics. M.S. received consultancy fees or honoraria from Abbott Vascular, AstraZeneca, Bayer, Boehringer Ingelheim, Merck Sharp \& Dohme, Novartis, and Vifor Pharma. S.J.S. received research funding from Actelion, the American Heart Association, AstraZeneca, Corvia, National Institutes of Health, and Novartis, and consulting fees from Actelion, Amgen, AstraZeneca, Bayer, Boehringer Ingelheim, Cardiora, Coridea, Eisai, Ionis, Ironwood, Merck, MyoKardia, Novartis, Sanofi, Tenax, and United Therapeutics. J.S. is an employee of and shareholder in Medtronic. S.S. is an employee of Huawei. R.v.L. is the founder and owner of McRoberts. H.D.D., C.J., and S.N. report no disclosures related to this paper.

\section{Funding Source}

This work was sponsored by Bayer AG.

\section{Author Contributions}

All authors read and approved the final manuscript. J.B., R.C., S.C., C.D., H.D.D., W.D., F.K., G.J.K., K.P., J.S., S.J.S., T.V.: study concept. J.B., L.B., P.C., R.C., H.D.D., W.D., P.C., C.G., C.J., F.K., A.M., S.N., A.P., P.P., S.R., J.S., M.S., S.S., S.J.S., R.v.L.: study investigation. W.D., F.K., T.K., J.S., S.S., R.v.L.: funding acquisition. J.B., H.D.D., W.D., F.K., S.J.S.: original draft development. P.C., C.D., C.G., T.K., A.M., A.P., S.S.: software development. W.D., F.K., G.J.K., K.P.: project administration.

\section{References}

1 Ponikowski P, Anker SD, AlHabib KF, Cowie MR, Force TL, Hu S, et al. Heart failure: preventing disease and death worldwide. ESC Heart Fail. 2014 Sep;1(1):4-25.

2 Savarese G, Lund LH. Global public health burden of heart failure. Card Fail Rev. 2017 Apr;3(1):7-11.

3 Owan TE, Hodge DO, Herges RM, Jacobsen SJ, Roger VL, Redfield MM. Trends in prevalence and outcome of heart failure with preserved ejection fraction. N Engl J Med. 2006 Jul;355(3):251-9.

4 Cleland JG, Tendera M, Adamus J, Freemantle N, Polonski L, Taylor J; PEP-CHF Investigators. The perindopril in elderly people with chronic heart failure (PEP-CHF) study. Eur Heart J. 2006 Oct;27(19):2338-45.

5 Pitt B, Pfeffer MA, Assmann SF, Boineau R, Anand IS, Claggett B, et al.; TOPCAT Investigators. Spironolactone for heart failure with preserved ejection fraction. N Engl J Med. 2014 Apr;370(15):1383-92.

6 van Veldhuisen DJ, Cohen-Solal A, Böhm M, Anker SD, Babalis D, Roughton M, et al.; SENIORS Investigators. Beta-blockade with nebivolol in elderly heart failure patients with impaired and preserved left ventricular ejection fraction: Data from SENIORS (Study of Effects of Nebivolol Intervention on Outcomes and Rehospitalization in Seniors with Heart Failure). J Am Coll Cardiol. 2009 Jun;53(23):2150-8.

7 Patel A, MacMahon S, Chalmers J, Neal B, Billot L, Woodward M, et al.; ADVANCE Collaborative Group. Intensive blood glucose control and vascular outcomes in patients with type 2 diabetes. N Engl J Med. 2008 Jun;358(24): 2560-72.

8 Kao DP, Lewsey JD, Anand IS, Massie BM, Zile MR, Carson PE, et al. Characterization of subgroups of heart failure patients with preserved ejection fraction with possible implications for prognosis and treatment response. Eur J Heart Fail. 2015 Sep;17(9):925-35. 
9 Shah RV, Desai AS, Givertz MM. The effect of renin-angiotensin system inhibitors on mortality and heart failure hospitalization in patients with heart failure and preserved ejection fraction: a systematic review and metaanalysis. J Card Fail. 2010 Mar;16(3):260-7.

10 Redfield MM, Anstrom KJ, Levine JA, Koepp GA, Borlaug BA, Chen HH, et al.; NHLBI Heart Failure Clinical Research Network. Isosorbide mononitrate in heart failure with preserved ejection fraction. N Engl J Med. 2015 Dec;373(24):2314-24.

11 Jaarsma T, Johansson P, Agren S, Strömberg A. Quality of life and symptoms of depression in advanced heart failure patients and their partners. Curr Opin Support Palliat Care. 2010 Dec;4(4):233-7.

12 Gwaltney CJ, Slagle AF, Martin M, Ariely R, Brede Y. Hearing the voice of the heart failure patient: key experiences identified in qualitative interviews. Br J Cardiol. 2012;19(1):25.

13 Psotka MA, von Maltzahn R, Anatchkova M, Agodoa I, Chau D, Malik FI, et al. Patient-reported outcomes in chronic heart failure: applicability for regulatory approval. JACC Heart Fail. 2016 Oct;4(10):791-804.

14 Demeyer H, Burtin C, Van Remoortel H, Hornikx M, Langer D, Decramer M, et al. Standardizing the analysis of physical activity in patients with COPD following a pulmonary rehabilitation program. Chest. 2014 Aug; 146(2):318-27.

15 [Internet] Qualification opinion on PROactive in COPD, 2018. [cited 31 May 2018]. Available from http:// www.ema.europa.eu/docs/en_GB/document_library/Regulatory_and_procedural_guideline/2018/04/ WC500247624.pdf

16 van Schooten KS, Rispens SM, Elders PJ, Lips P, van Dieën JH, Pijnappels M. Assessing physical activity in older adults: required days of trunk accelerometer measurements for reliable estimation. J Aging Phys Act. 2015 Jan;23(1):9-17.

17 Green CP, Porter CB, Bresnahan DR, Spertus JA. Development and evaluation of the Kansas City Cardiomyopathy Questionnaire: a new health status measure for heart failure. J Am Coll Cardiol. 2000 Apr;35(5):124555.

18 Faggiano P, D'Aloia A, Gualeni A, Brentana L, Dei Cas L. The 6 minute walking test in chronic heart failure: indications, interpretation and limitations from a review of the literature. Eur J Heart Fail. 2004 Oct;6(6): 687-91.

19 Guyatt GH, Pugsley SO, Sullivan MJ, Thompson PJ, Berman L, Jones NL, et al. Effect of encouragement on walking test performance. Thorax. 1984 Nov;39(11):818-22.

20 ATS Committee on Proficiency Standards for Clinical Pulmonary Function Laboratories. ATS statement: guidelines for the six-minute walk test. Am J Respir Crit Care Med. 2002 Jul;166(1):111-7.

21 Feng J, Schaus BJ, Fallavollita JA, Lee TC, Canty JM Jr. Preload induces troponin I degradation independently of myocardial ischemia. Circulation. 2001 Apr;103(16):2035-7.

22 Cardinale D, Colombo A, Sandri MT, Lamantia G, Colombo N, Civelli M, et al. Prevention of high-dose chemotherapy-induced cardiotoxicity in high-risk patients by angiotensin-converting enzyme inhibition. Circulation. 2006 Dec;114(23):2474-81.

23 Latini R, Masson S, Anand IS, Missov E, Carlson M, Vago T, et al.; Val-HeFT Investigators. Prognostic value of very low plasma concentrations of troponin $\mathrm{T}$ in patients with stable chronic heart failure. Circulation. 2007 Sep;116(11):1242-9.

24 Santhanakrishnan R, Chong JP, Ng TP, Ling LH, Sim D, Leong KT, et al. Growth differentiation factor 15, ST2, high-sensitivity troponin $\mathrm{T}$, and $\mathrm{N}$-terminal pro brain natriuretic peptide in heart failure with preserved vs. reduced ejection fraction. Eur J Heart Fail. 2012 Dec;14(12):1338-47.

25 Kramer F. Galectin-3: clinical utility and prognostic value in patients with heart failure. Res Rep Clin Cardiol. 2013;4:13-22.

26 Kempf T, Eden M, Strelau J, Naguib M, Willenbockel C, Tongers J, et al. The transforming growth factor-beta superfamily member growth-differentiation factor-15 protects the heart from ischemia/reperfusion injury. Circ Res. 2006 Feb;98(3):351-60.

27 Wang F, Guo Y, Yu H, Zheng L, Mi L, Gao W. Growth differentiation factor 15 in different stages of heart failure: potential screening implications. Biomarkers. 2010 Dec;15(8):671-6.

28 Kempf T, von Haehling S, Peter T, Allhoff T, Cicoira M, Doehner W, et al. Prognostic utility of growth differentiation factor-15 in patients with chronic heart failure. J Am Coll Cardiol. 2007 Sep;50(11):1054-60.

29 Weinberg EO, Shimpo M, Hurwitz S, Tominaga S, Rouleau JL, Lee RT. Identification of serum soluble ST2 receptor as a novel heart failure biomarker. Circulation. 2003 Feb;107(5):721-6.

30 Wang YC, Yu CC, Chiu FC, Tsai CT, Lai LP, Hwang JJ, et al. Soluble ST2 as a biomarker for detecting stable heart failure with a normal ejection fraction in hypertensive patients. J Card Fail. 2013 Mar;19(3):163-8.

31 Ky B, French B, McCloskey K, Rame JE, McIntosh E, Shahi P, et al. High-sensitivity ST2 for prediction of adverse outcomes in chronic heart failure. Circ Heart Fail. 2011 Mar;4(2):180-7.

32 Maisel AS, Krishnaswamy P, Nowak RM, McCord J, Hollander JE, Duc P, et al.; Breathing Not Properly Multinational Study Investigators. Rapid measurement of B-type natriuretic peptide in the emergency diagnosis of heart failure. N Engl J Med. 2002 Jul;347(3):161-7.

33 Lasota B, Mizia-Stec K. Copeptin in heart failure. Res Rep Clin Cardiol. 2014;5:133-44.

34 Neuhold S, Huelsmann M, Strunk G, Stoiser B, Struck J, Morgenthaler NG, et al. Comparison of copeptin, B-type natriuretic peptide, and amino-terminal pro-B-type natriuretic peptide in patients with chronic heart failure: prediction of death at different stages of the disease. J Am Coll Cardiol. 2008 Jul;52(4):266-72. 
35 Maisel A, Xue Y, Shah K, Mueller C, Nowak R, Peacock WF, et al. Increased 90-day mortality in patients with acute heart failure with elevated copeptin: secondary results from the Biomarkers in Acute Heart Failure (BACH) study. Circ Heart Fail. 2011 Sep;4(5):613-20.

36 Loncar G, von Haehling S, Tahirovic E, Inkrot S, Mende M, Sekularac N, et al. Effect of beta blockade on natriuretic peptides and copeptin in elderly patients with heart failure and preserved or reduced ejection fraction: results from the CIBIS-ELD trial. Clin Biochem. 2012 Jan;45(1-2):117-22.

37 Barroso MC, Kramer F, Greene SJ, Scheyer D, Köhler T, Karoff M, et al. Serum insulin-like growth factor-1 and its binding protein-7: potential novel biomarkers for heart failure with preserved ejection fraction. BMC Cardiovasc Disord. 2016 Oct;16(1):199.

38 Chugh S, Ouzounian M, Lu Z, Mohamed S, Li W, Bousette N, et al. Pilot study identifying myosin heavy chain 7, desmin, insulin-like growth factor 7 , and annexin A2 as circulating biomarkers of human heart failure. Proteomics. 2013 Aug;13(15):2324-34.

39 Gottlieb S. Gottlieb admonishes industry for clinical trial failings. 2019 Available from: http://www.pmlive. com/pharma_news/gottlieb_admonishes_industry_for_clinical_trial_failings_1281687. Accessed: 20 March 2019.

40 Warman EN, Grammatico A, Padeletti L. Sample size estimates for atrial fibrillation endpoints. Heart Rhythm. 2004 Jul;1(2 Suppl):B58-62.

41 Ponikowski P, Voors AA, Anker SD, Bueno H, Cleland JG, Coats AJ, et al.; Authors/Task Force Members; Document Reviewers. 2016 ESC Guidelines for the diagnosis and treatment of acute and chronic heart failure: the Task Force for the diagnosis and treatment of acute and chronic heart failure of the European Society of Cardiology (ESC). Developed with the special contribution of the Heart Failure Association (HFA) of the ESC. Eur J Heart Fail. 2016 Aug;18(8):891-975.

42 Nadruz W Jr, West E, Santos M, Skali H, Groarke JD, Forman DE, et al. Heart failure and midrange ejection fraction: implications of recovered ejection fraction for exercise tolerance and outcomes. Circ Heart Fail. 2016 Apr;9(4):e002826.

43 Gheorghiade M, Vaduganathan M, Fonarow GC, Bonow RO. Rehospitalization for heart failure: problems and perspectives. J Am Coll Cardiol. 2013 Jan;61(4):391-403.

44 Anand IS, Tang WH, Greenberg BH, Chakravarthy N, Libbus I, Katra RP; Music Investigators. Design and performance of a multisensor heart failure monitoring algorithm: results from the multisensor monitoring in congestive heart failure (MUSIC) study. J Card Fail. 2012 Apr;18(4):289-95.

45 Innovative Medicines Initiative. Linking digital assessment of mobility to clinical endpoints to support regulatory acceptance and clinical practice. 2017; Available from http://ec.europa.eu/research/participants/ portal/desktop/en/opportunities/h2020/topics/imi2-2017-13-07.html. Accessed 12 July 2018. 